\title{
Large-scale photospheric dynamics below coronal holes
}

\author{
N. Meunier \\ Laboratoire d'Astrophysique de l'Observatoire Midi-Pyrénées, 57 avenue d'Azereix, BP 826, 65008 Tarbes Cedex, France \\ e-mail: meunier@bagn.obs-mip.fr
}

Received 15 April 2005 / Accepted 11 July 2005

\section{ABSTRACT}

A large data set of MDI magnetograms is studied to analyze the global dynamics of magnetic structures from network to active regions inside and outside coronal holes. We find a weaker meridional circulation at almost all latitudes, as well as a more differential rotation of the photospheric magnetic structures below high-latitude coronal holes. Stronger asymmetries in the dynamics are observed in coronal holes at high latitude compared to outside coronal holes. The variation in the dynamics is also studied as a function of the coronal hole size as well as its variation with time and with magnetic field. The smaller velocity dispersion obtained in coronal holes when using a cross-correlation technique shows that the large-scale circulation may be inhibited inside coronal holes compared to outside. However, there is an indication of a larger diffusion of magnetic features within coronal holes than outside.

Key words. Sun: magnetic fields - Sun: photosphere - Sun: activity - Sun: corona

\section{Introduction}

Coronal holes (herefafter $\mathrm{CHs}$ ) are defined as regions of open magnetic field lines (Wang et al. 1996), as opposed to closed loops mostly associated with active regions. They are observed as dark areas in X-ray and EUV, and as bright regions in spectroheliograms made in the He I $\lambda 10830 \AA$ line. They are also associated with regions that are almost unipolar in the photosphere. Identification procedures for $\mathrm{CHs}$ have been elaborated using complementary data sets, for example by Harvey \& Recely (2002) who used a combination of He I spectroheliograms and photospheric magnetograms. There are three types of CHs: polar $\mathrm{CHs}$, low latitude extension of polar $\mathrm{CHs}$ and transient $\mathrm{CHs}$ more closely associated with active regions. Their respective typical lifetimes are very variable, as is their distribution in time over the solar cycle (e.g. Harvey \& Recely 2002).

However, all properties of $\mathrm{CHs}$ are not known nor understood. For example, not all unipolar regions are associated with a $\mathrm{CH}$, and the differences between similarly quiet photospheric regions in and outside $\mathrm{CHs}$ are not well known (De Toma \& Arge 2005), despite studies of the magnetic properties inside and outside CHs (Harvey et al. 1982; Harvey \& Recely 2002). Also, polar CHs with extensions towards low latitudes exhibit a rigid rotation, despite the differential rotation of the magnetic structures in the photosphere (Snodgrass \& Ulrich 1990; Komm et al. 1993a; Meunier 1999, 2005a) and of the coronal bright points (Antonucci et al. 1979; Brajša et al. 2004). There have been several theoretical works (e.g. Wang \& Sheeley 1990, 2004; Fisk et al. 1999) to explain this rigid rotation, which is likely due to processes occuring at the boundary of CHs. The study of the boundaries of $\mathrm{CHs}$ is therefore very important. Kahler \& Hudson (2002) have studied in detail the morphology of the boundary of long-lived coronal holes. However, we are still lacking observations of the dynamics in the photosphere in relation to $\mathrm{CHs}$.

In this paper, the large-scale photospheric dynamics of small magnetic features is determined for the first time inside and outside CHs. The observations and data analysis are described in Sect. 2. The resulting meridional circulation and angular rotation velocity, hereafter rotation rate, are presented in Sect. 3. Additional information can be derived from the dispersion in these velocities. The results are discussed in Sect. 4.

\section{Observations and data analysis}

\subsection{MDI magnetograms}

Full-disk magnetograms obtained by MDI/SOHO (Scherrer et al. 1995) cover most of cycle 23. In this study, 5-min averaged magnetograms ${ }^{1}$ at level 1.8 , i.e. the latest data level provided by the MDI/SOHO team, are used between June 1996 and September 2003. The averaged magnetograms are separated by $96 \mathrm{~min}$ or a multiple of $96 \mathrm{~min}$, with a few gaps (see Meunier 2005b, for the temporal distribution of pairs of magnetograms). Only pairs of magnetograms separated by $96 \mathrm{~min}$ are considered in this study.

Large-scale dynamics using correlation tracking (hereafter CT) are derived from these pairs of magnetograms separated by $96 \mathrm{~min}$ as described in Meunier (2005a), with a method

\footnotetext{
${ }^{1}$ An averaged magnetogram is computed from 5 single magnetograms covering $5 \mathrm{~min}$.
} 
similar to that of Komm et al. (1993a,b). The correlation tracking is done by cross-correlating boxes of size $7.68 \times 7.68 \mathrm{deg}^{2}$ $\left(93 \times 93 \mathrm{Mm}^{2}\right)$ on pairs of magnetograms after remapping on a grid with constant steps in latitude and longitude, as in Meunier (1999). The average unsigned magnetic field $\left\langle\left|B_{\mathrm{c}}\right|\right\rangle$ is computed over each box, as well as the unsigned maximum magnetic field $\left\langle\left|B_{\mathrm{mc}}\right|\right\rangle$. The output of CT consists of maps of horizontal velocities (rotation and meridional circulation). The comparison with the large-scale dynamics of individual features obtained by feature tracking (hereafter FT), also described in Meunier (2005a), has also been attempted. Magnetic structures are defined by adjacent pixels with a magnetic field above a threshold of $40 \mathrm{G}$ (or below $-40 \mathrm{G}$ for negative magnetic fields). These structures are then tracked between pairs of magnetograms separated by $96 \mathrm{~min}$ as for CT. The output of FT is a list of structures associated with their properties (such as size or average magnetic field) and velocities (rotation and meridional circulation as for CT).

\subsection{The coronal hole data set}

Coronal hole boundaries over a long period have been determined using Kitt Peak full-disk photospheric magnetograms and He I spectroheliograms (Harvey \& Recely 2002). Figure 1 shows an example of such boundaries. The coordinates of the boundaries have been retrieved from the archive at $\mathrm{ftp}: / /$ solarch.tuc.noao.edu/kpvt/coronal_holes/. They are available with a $1^{\circ}$ spatial resolution in latitude and longitude.

Due to possible gaps in the $\mathrm{CH}$ contours, the original resolution of $1^{\circ}$ has been degraded by a factor of 2 to avoid losing too many $\mathrm{CH}$ data points. Low resolution maps $\left(2^{\circ}\right)$ are therefore derived from these boundaries (see an example in Fig. 1). The areas occupied by the $\mathrm{CHs}$ are determined from these boundaries. This analysis provides 3 types of pixels:

- pixels outside CHs have 0 values;

- pixels inside CHs have positive values;

- boundary pixels have negative values.

Because of the low resolution, small CHs or thin extensions of $\mathrm{CHs}$ have negative values only, i.e. as if they were only constituted of boundaries. Therefore boundary $\mathrm{CH}$ pixels correspond either to an actual boundary pixel of a large $\mathrm{CH}$ or to a small $\mathrm{CH}$ including the core of the $\mathrm{CH}$. In the following, three categories of $\mathrm{CH}$ regions will be studied: inside $\mathrm{CHs}$ (positive values), $\mathrm{CH}$ boundary (negative values), all $\mathrm{CHs}$ (both positive and negative values). For each CT box (or each structure defined by FT), the location on the low resolution map is identified after correction of their rotation $(\mathrm{CH}$ maps are available once per day or less often and MDI data are available a few time per day at most). The number of boxes or features for each category is shown in Table 1 . CT results for which $\mathrm{CH}$ data are not available are of course eliminated from the analysis.

\subsection{Selection biases}

Because CHs are unevenly distributed over the solar surface, great care must be taken to possible selection effects when comparing the dynamics inside and outside CHs. Similarly,
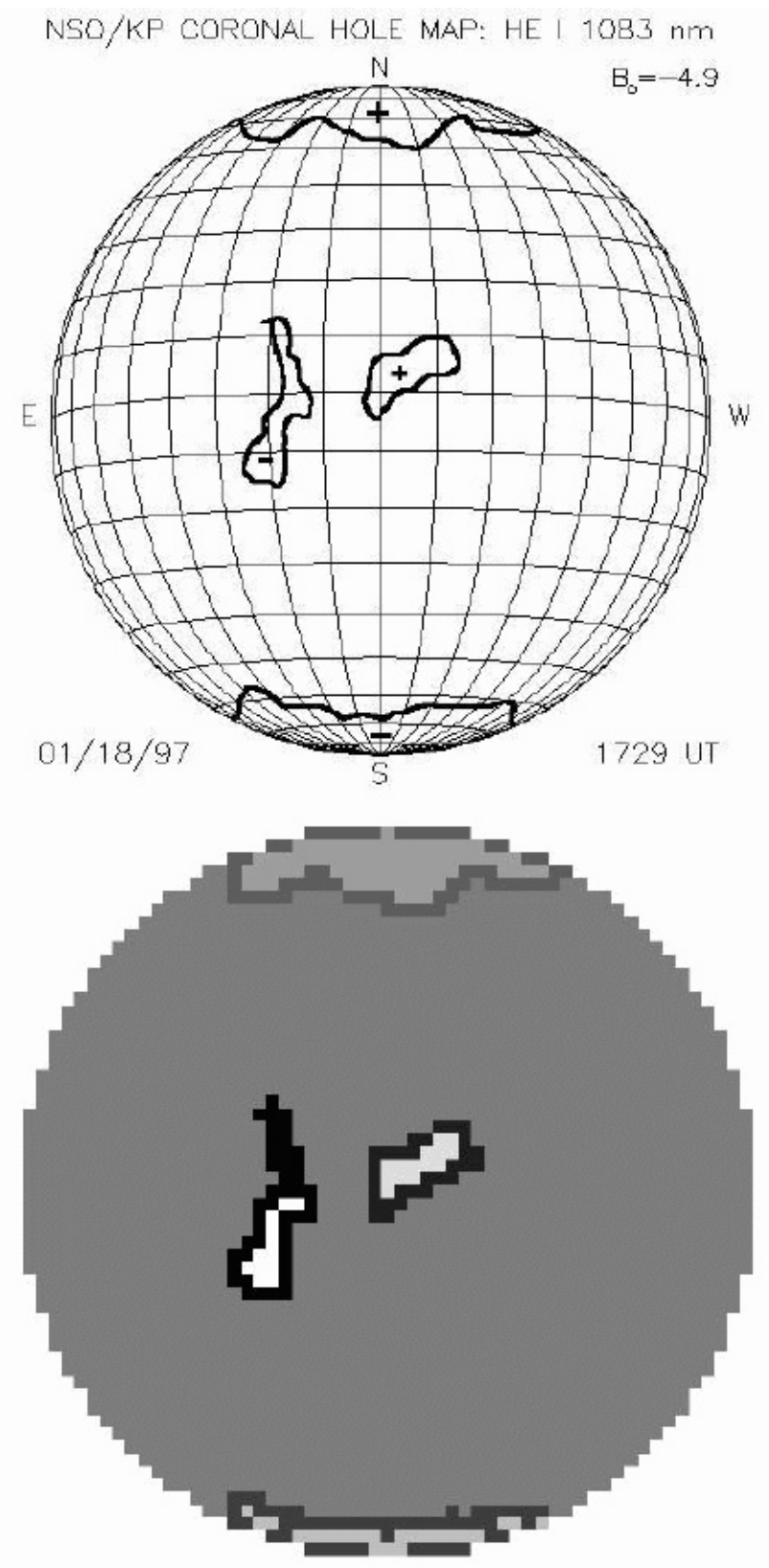

Fig. 1. Top: coronal hole contour for 1997 January 18 (plot from the Kitt Peak archive). Bottom: low resolution map derived from the list of coordinates of the coronal hole boundaries. $\mathrm{CH}$ boundary pixels are the darker ones, and inside $\mathrm{CH}$ pixels are the brighter ones.

Table 1. Number of data points for each category, before selection (all) and after selection (sel), as described in Sect. 2.3.

\begin{tabular}{ccccc}
\hline \hline & all CT & sel CT & all FT & sel FT \\
\hline Outside CHs & 1284090 & 442231 & 593084 & 181325 \\
Inside CHs & 38167 & 19131 & 16440 & 5749 \\
CH boundary & 379247 & 87929 & 29857 & 9956 \\
\hline
\end{tabular}

the number of $\mathrm{CHs}$ varies during the solar cycle and selection biases are possible. Furthermore, the magnetic level inside and outside coronal holes is different, as active regions are not present inside CHs. Figure 2 shows the distribution of 

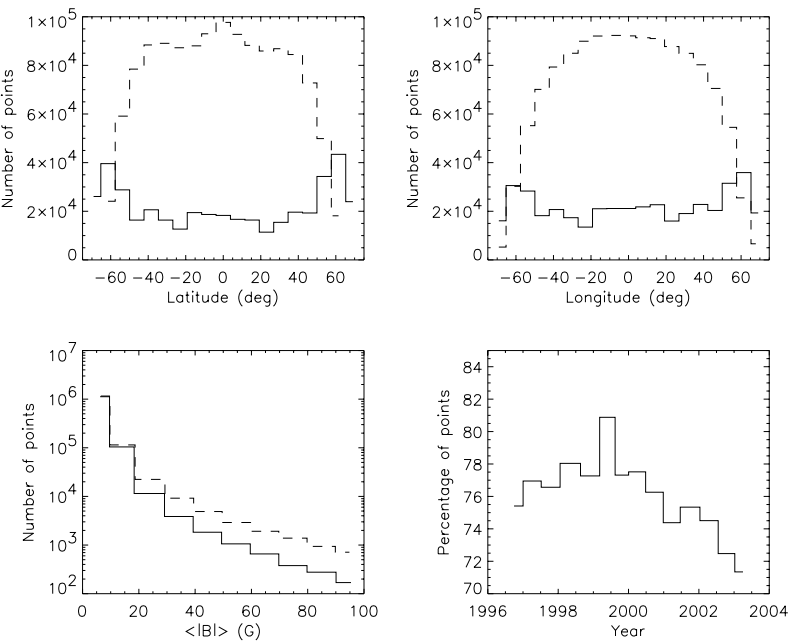

Fig. 2. Top left: number of CT points outside CHs (dashed line) and for all $\mathrm{CHs}$ (solid line) versus latitude. Top right: same plot versus longitude. Bottom right: same plot versus $\left\langle\left|B_{\mathrm{c}}\right|\right\rangle$ inside the CT boxes. Bottom left: percentage of points outside $\mathrm{CHs}$.

the number of $\mathrm{CT}$ boxes outside $\mathrm{CHs}$ and for all $\mathrm{CH}$ data versus latitude, longitude, time and $\left\langle\left|B_{\mathrm{c}}\right|\right\rangle$. Because the latitudinal and temporal variations are large, the variation in the dynamics with time and latitude will be studied in the following. Furthermore, the distribution versus longitude is very different for points outside and in CHs. Therefore, in the following, points within $\pm 16^{\circ}$ of the central meridian only are selected. For the same reason, only weak field regions will be considered, namely with $\left\langle\left|B_{\mathrm{c}}\right|\right\rangle$ lower than $20 \mathrm{G}$ for $\mathrm{CT}$, and sizes below $100 \mathrm{Mm}^{2}$ for FT (i.e. network structures, see Wang 1988; and Meunier 2003). When using slightly different thresholds, the results presented in Sect. 3 are not significantly affected, because they are dominated by the weakest field regions. The number of boxes or features for each category after this selection is shown in Table 1.

\subsection{A few $\mathrm{CH}$ properties}

Figure 3 shows that the distributions of the average unsigned magnetic field $\left\langle\left|B_{\mathrm{c}}\right|\right\rangle$ and maximum unsigned magnetic field $\left\langle\left|B_{\mathrm{mc}}\right|\right\rangle$ in $\mathrm{CT}$ boxes are quite similar inside $\mathrm{CHs}$ and at the $\mathrm{CH}$ boundary. The only exception is an excess of points for $\left\langle\left|B_{\mathrm{mc}}\right|\right\rangle$ in the range 50-400 $\mathrm{G}$ inside $\mathrm{CHs}$ compared to the distribution in $\left\langle\left|B_{\mathrm{c}}\right|\right\rangle$ and to what is observed for the $\mathrm{CH}$ boundary. Figure 3 shows that the distributions outside $\mathrm{CHs}$ extend toward much larger magnetic fields due to the presence of active regions.

Figure 4 shows the variation with time in average unsigned magnetic field outside $\mathrm{CHs}$, inside $\mathrm{CHs}$ and at $\mathrm{CH}$ boundary before and after the $\left\langle\left|B_{\mathrm{c}}\right|\right\rangle$ selection described in Sect. 2.3. In the following, cycle minimum corresponds to the period until June 1997 and cycle maximum cover the period from May 1999 to December 2003. In the first case, the ratio between the average $\left\langle\left|B_{\mathrm{c}}\right|\right\rangle$ at cycle maximum and at cycle minimum is respectively $1.80,1.43$ and 1.20 . After the selection $\left\langle\left|B_{\mathrm{c}}\right|\right\rangle<$ $20 \mathrm{G}$, they are $1.41,1.38$ and 1.14 . On the other hand, the flux inside $\mathrm{CHs}$ is known to be variable during the solar cycle.
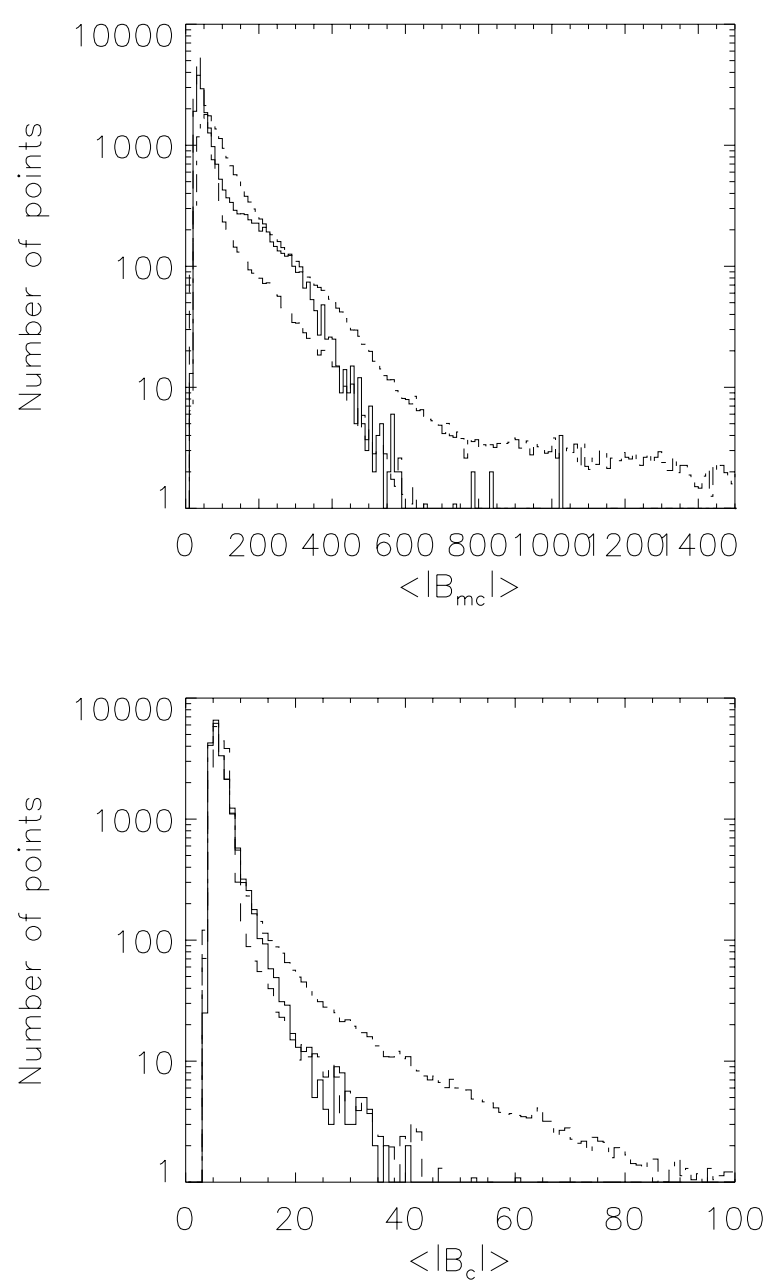

Fig. 3. Top: distribution of the maximum unsigned magnetic field $\left\langle\left|B_{\text {mc }}\right|\right\rangle$ for regions outside $\mathrm{CHs}$ (dotted-dashed line), inside CHs (solid line) and at the $\mathrm{CH}$ boundary (dashed line). Histograms are normalized to the same number of points. Bottom: same for the average unsigned magnetic field $\left\langle\left|B_{\mathrm{c}}\right|\right\rangle$. All plots are for CT boxes within $\pm 16^{\circ}$ of the central meridian.

Harvey et al. (1982) found a flux 3 times larger at cycle maximum than at cycle minimum inside low latitude $\mathrm{CHs}$ (for a similar area), which means that the average magnetic field is increasing by the same factor. In our case, we find a smaller variation in the average magnetic field inside $\mathrm{CHs}$ compared to outside (top of Fig. 4). This could be due to a selection effect when computing the dynamics. The dynamics are indeed not computed everywhere on the disk but only when the signal is large enough. In practice, the cross-correlation with amplitude lower than 0.2 are eliminated in the analysis. As a consequence, the weakest field regions are not counted, which has two effects. The first is that the average magnetic field is overestimated. This could be different inside $\mathrm{CHs}$ and outside $\mathrm{CHs}$ if the proportion of very weak field regions is different, as well as between cycle minimum and cycle maximum for the same reason. Therefore it is quite likely that the ratio is underestimated in both cases, especially in the case of $\mathrm{CHs}$ where we expect more weak field regions. Second, the proportion of the surface occupied by $\mathrm{CHs}$ is also different from what is usually observed. G. de Toma (private communication) has estimated 

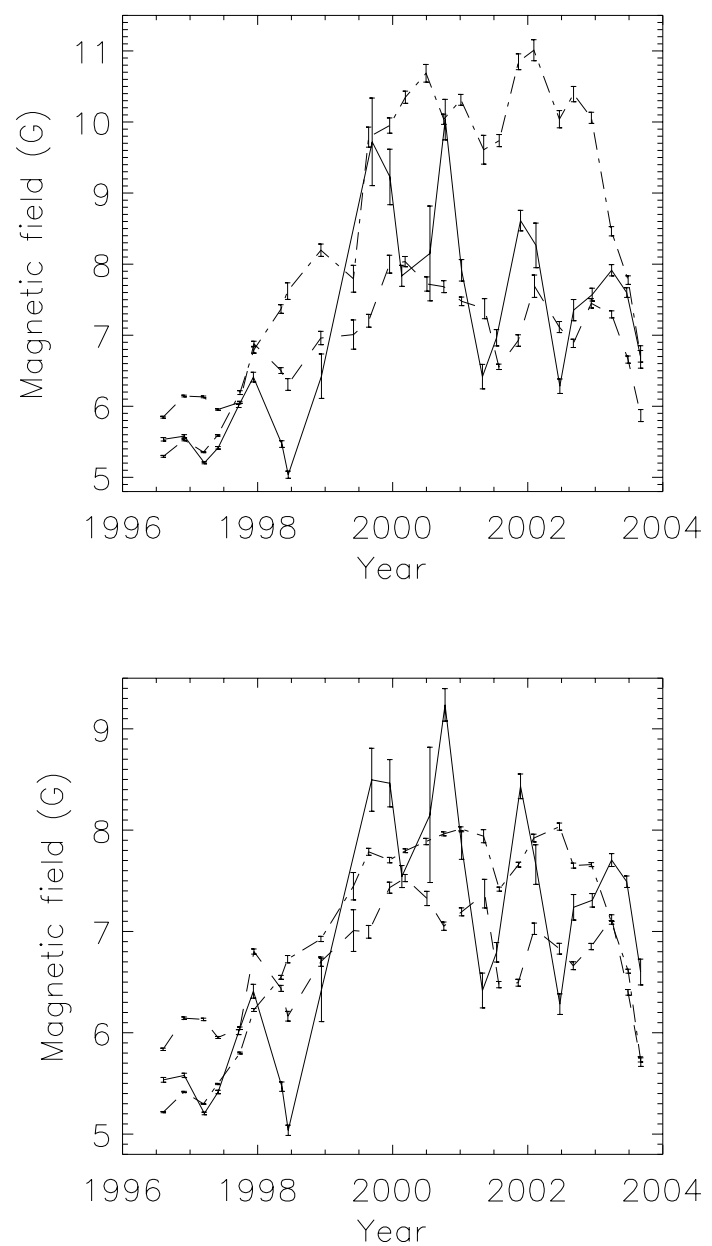

Fig. 4. Top: variation in $\left\langle\left|B_{\mathrm{c}}\right|\right\rangle$ outside $\mathrm{CHs}$ (dotted-dashed line), inside $\mathrm{CHs}$ (solid line) and at $\mathrm{CH}$ boundary (line) with time for all $\mathrm{CT}$ boxes. Bottom: same for CT boxes with $\left\langle\left|B_{\mathrm{c}}\right|\right\rangle$ lower than $20 \mathrm{G}$. All plots are for CT boxes within $\pm 16^{\circ}$ of the central meridian.

a coverage of about $20-30 \%$ of the surface by $\mathrm{CHs}$ at cycle minimum (from the same $\mathrm{CH}$ data base) and a coverage lower than $5 \%$ at cycle maximum. Here the number of points inside $\mathrm{CHs}$ and at the $\mathrm{CH}$ boundary is about $22 \%$ at cycle minimum (after selection) and still 18\% at cycle maximum. The proportions are very similar before the selection described in Sect. 2.3, so the sampling effect related to the discarding of regions with low cross-correlation could explain this difference.

Figure 5 shows the $\mathrm{CH}$ size distribution for various latitude ranges in our CT sample. There is an asymmetry between hemispheres at high latitudes. The size distributions at high latitude show a peak around 5-6\% of the visible hemisphere. On the other hand, at low latitude, there is a strong peak for small size (below $3 \%$ of the visible hemisphere), as well as a significant contribution in the range $5-6 \%$ as well, which may be due to the fact that a large fraction of high latitude CHs have an extension toward low latitudes. There is also an asymmetry between hemispheres at low latitudes.

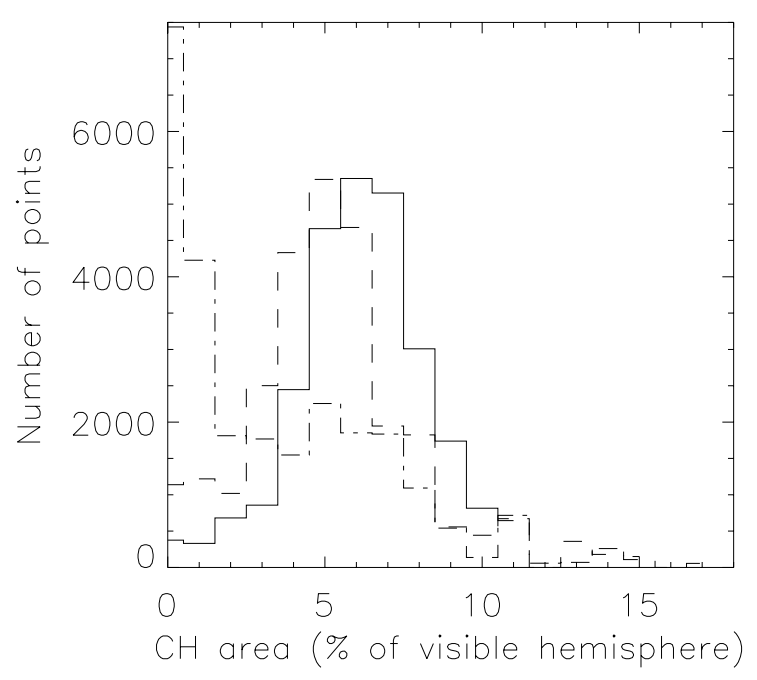

Fig. 5. $\mathrm{CH}$ area distribution above latitude $40^{\circ}$ (solid line), below latitude $-40^{\circ}$ (dashed line), and between latitudes $-40^{\circ}$ and $40^{\circ}$ (dotteddashed line). Plots are for CT boxes within $\pm 16^{\circ}$ of the central meridian and for CT boxes with $\left\langle\left|B_{\mathrm{c}}\right|\right\rangle$ lower than $20 \mathrm{G}$.

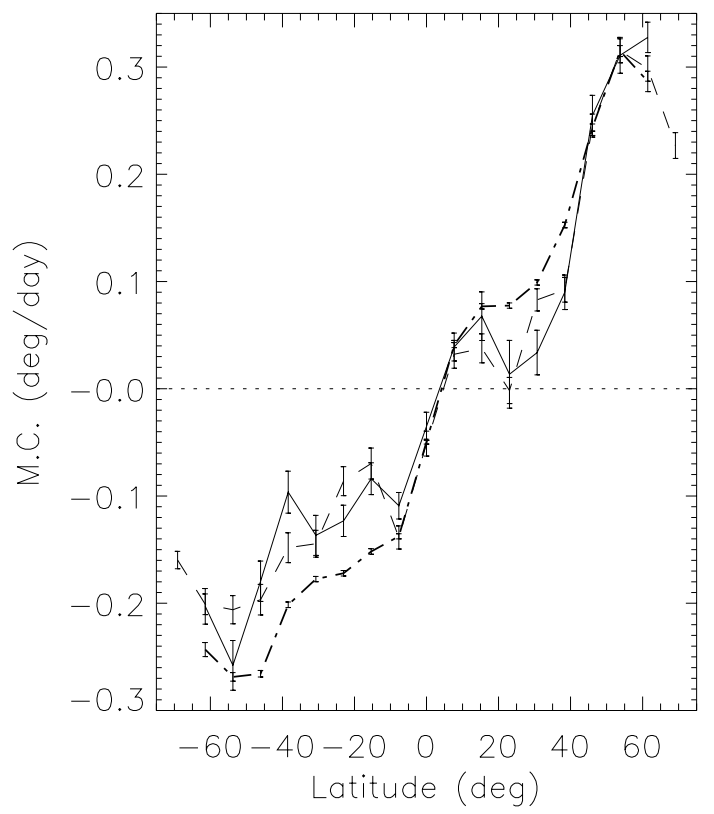

Fig. 6. Meridional circulation versus latitude outside $\mathrm{CHs}$ (thick dotted-dashed line), inside $\mathrm{CHs}$ (solid line), and at the $\mathrm{CH}$ boundary (dashed line). Positive meridional circulation is toward the North pole. Plots are for $\mathrm{CT}$ boxes within $\pm 16^{\circ}$ of the central meridian and for $\mathrm{CT}$ boxes with $\left\langle\left|B_{\mathrm{c}}\right|\right\rangle$ lower than $20 \mathrm{G}$.

\section{Results}

\subsection{Meridional circulation}

Figure 6 shows the meridional circulation versus latitude for the various categories. In the range $10-40^{\circ}$, i.e. in the activity belt, the meridional circulation is less poleward inside $\mathrm{CHs}$ and at the $\mathrm{CH}$ boundary than outside $\mathrm{CH}$ in both hemispheres. Meunier (1999, 2005a) for example observed a converging pattern (higher poleward motion at low latitude compared to the adjacent poleward latitude band) in the activity belt superimposed on the traditional poleward meridional circulation 

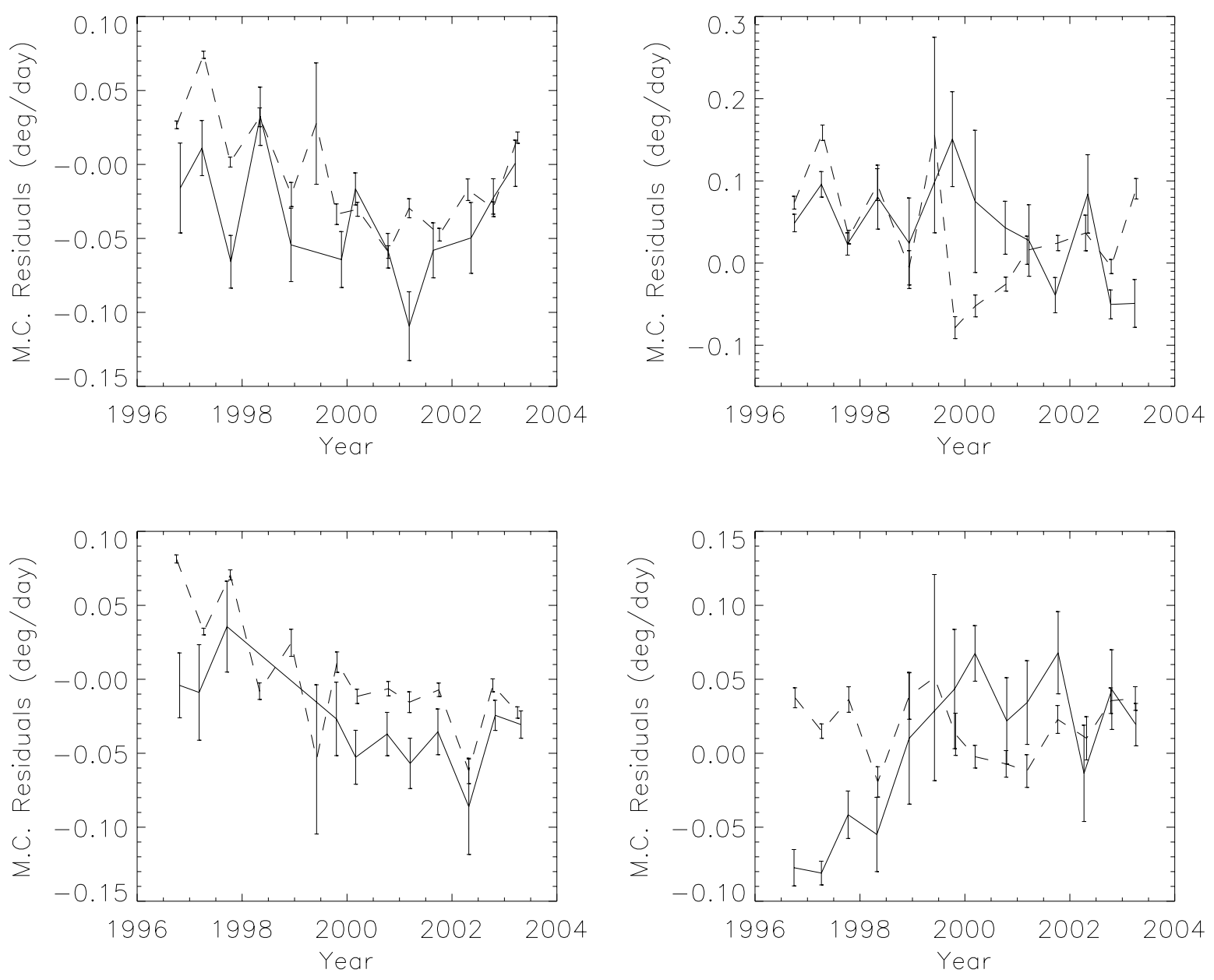

Fig. 7. Meridional circulation residuals versus time outside CHs (dashed line) and for all CHs (solid line). Top: northern hemisphere. Bottom: southern hemisphere. Left: latitudes lower than $40^{\circ}$. Right: latitudes larger than $40^{\circ}$. Positive residuals correspond to a more poleward motion than average. Plots are for CT boxes within $\pm 16^{\circ}$ of the central meridian and for CT boxes with $\left\langle\left|B_{\mathrm{c}}\right|\right\rangle$ lower than $20 \mathrm{G}$.

which increases monotonously as a function of latitude (see also Haber et al. 2003; Zhao \& Kosovichev 2004). Meunier (1999) found that this pattern was mainly seen in active regions. The more precise study of Meunier (2005a) showed that this pattern was more pronounced for larger values of $\left\langle\left|B_{\mathrm{c}}\right|\right\rangle$ while it disappeared for very small values of $\left\langle\left|B_{\mathrm{c}}\right|\right\rangle$. Here, the converging pattern is clearly seen in $\mathrm{CH}$ in the weak field regime, but it is almost absent outside the $\mathrm{CH}$ for similar values of $\left\langle\left|B_{\mathrm{c}}\right|\right\rangle$. It also shows that this pattern is not associated with active regions only. At high latitude, i.e. above $40^{\circ}, \mathrm{CH}$ points have a smaller meridional circulation than outside $\mathrm{CH}$ in the Southern hemisphere. However, in the Northern hemisphere, the meridional circulation is very similar outside and inside $\mathrm{CHs}$, with a tendency for a slightly larger meridional circulation in $\mathrm{CHs}$. Therefore, the high-latitude asymmetry between hemispheres is larger in CHs than outside CHs.

The meridional circulation residuals are computed as in Meunier (2005a) as follows. The average meridional circulation in a latitude bin is subtracted from all values corresponding to that range. The sign is changed in the Southern hemisphere, so that a positive residuals means a more poleward motion than average, for that latitude range. Furthermore, Fig. 7 shows the meridional circulation residuals separately at high and low latitudes, and in the two hemispheres. At low latitude, the residuals in $\mathrm{CHs}$ are smaller than outside $\mathrm{CHs}$ at all time, which shows that the characteristics seen in Fig. 6 are quite stable. At high latitude on the other hand, the residuals are similar in and outside $\mathrm{CHs}$ at all time, with a tendency to have smaller residuals in CHs until 1999, larger ones from 2000 to 2001, and smaller ones afterwards. In the Southern hemisphere the tendency is the same with smaller errorbars, as the residuals are significantly smaller in $\mathrm{CHs}$ during the first years.

Figure 8 shows the average meridional circulation inside $\mathrm{CHs}$ versus the size of $\mathrm{CHs}$ in two cases, at high latitude and in the activity belt. At high latitude, there is a tendency for a smaller meridional circulation associated with small $\mathrm{CHs}$ in the Northern hemisphere but this is not seen in the Southern hemisphere. On the other hand, the weaker meridional circulation in CHs compared to outside is quite clear in the Southern hemisphere for almost all size ranges, except perhaps the very large ones. However, this is not the case in the Northern hemisphere, as the weaker meridional circulation seems only present for small CHs. Therefore, there is a strong asymmetry between hemispheres as already seen in Fig. 6. In the activity belt, the weaker meridional circulation in CHs is clearly seen for areas up to $7 \%$ of visible hemisphere. Above this value, it may not 

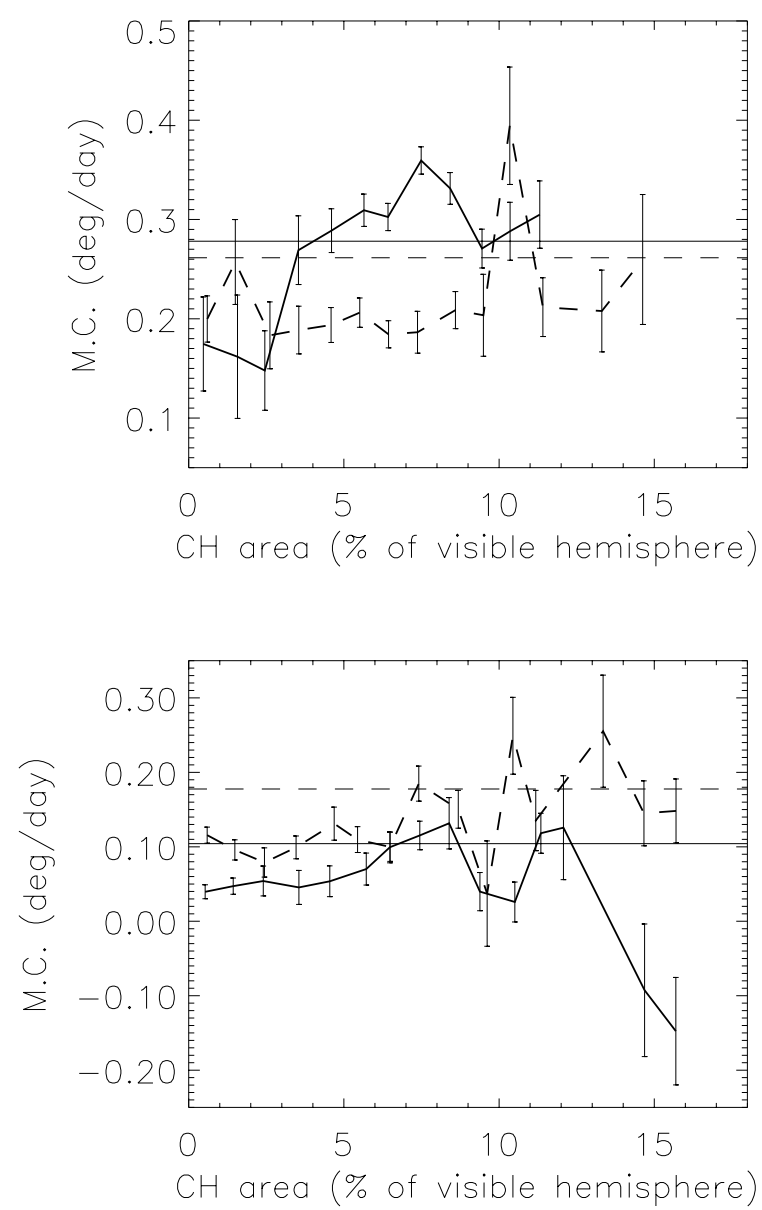

Fig. 8. Top: meridional circulation versus the $\mathrm{CH}$ size (extracted from the $\mathrm{CH}$ data base) at latitudes larger than $40^{\circ}$ in the Northern hemisphere (thick solid line) and in the Southern hemisphere (thick dashed line), for all $\mathrm{CH}$ categories. The horizontal thin lines correspond to the meridional circulation outside CHs. Bottom: same for the latitude range $10-40^{\circ}$. Positive meridional circulation is toward the poles. $\mathrm{CH}$ areas are in $\%$ of the visible hemisphere. Plots are for CT boxes within $\pm 16^{\circ}$ of the central meridian and for CT boxes with $\left\langle\left|B_{\mathrm{c}}\right|\right\rangle$ lower than $20 \mathrm{G}$.

be present, but the very large $\mathrm{CHs}$ in the Northern hemisphere may have a much smaller meridional circulation as well.

The variation in meridional circulation with increasing $\left\langle\left|B_{\mathrm{c}}\right|\right\rangle$ observed by Meunier (2005a) is computed separately for the different categories. The resulting decrease is shown in Fig. 9. For the weakest fields the slope is steeper inside $\mathrm{CHs}$ and at $\mathrm{CH}$ boundary than outside $\mathrm{CHs}$, up to $\left\langle\left|B_{\mathrm{c}}\right|\right\rangle \sim 6 \mathrm{G}$. For $\left\langle\left|B_{\mathrm{c}}\right|\right\rangle$ above $5 \mathrm{G}$, the meridional circulation become less poleward in CHs. Above $7 \mathrm{G}$, there is no significant difference as the uncertainties become larger.

The errorbars, larger in the FT method, prevented the detection of any difference of the order of those detected with CT when looking at the latitude variation with this technique.

\subsection{Rotation}

Table 2 shows the coefficients obtained from fits made with Legendre polynomials in $\sin ^{i}(\theta)$ ( $\theta$ the latitude) on the CT angular velocity versus latitude (as in Meunier 2005a).

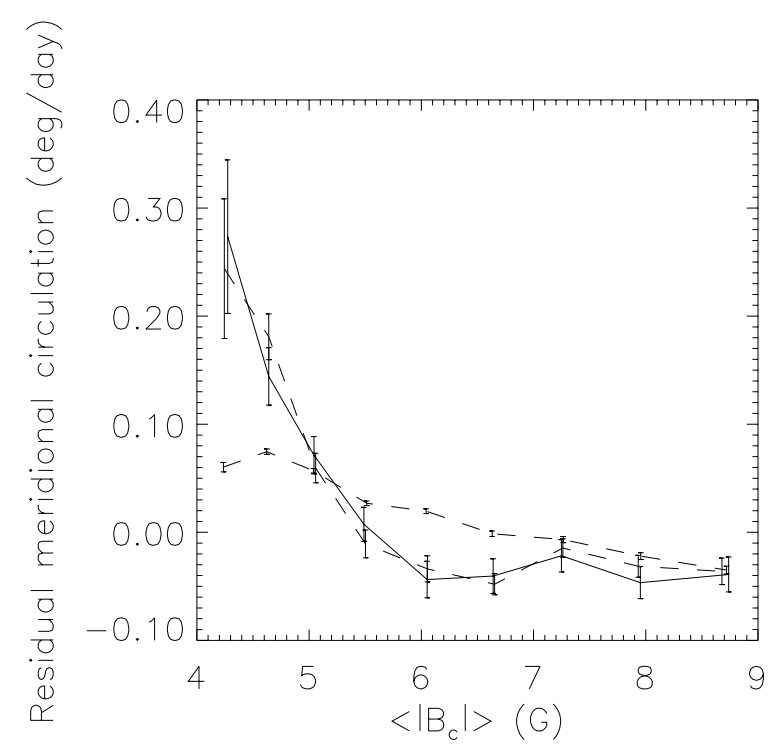

Fig. 9. Meridional circulation residuals versus $\left\langle\left|B_{\mathrm{c}}\right|\right\rangle$ outside $\mathrm{CH}$ (dotted-dashed line), inside $\mathrm{CHs}$ (solid line) and at the $\mathrm{CH}$ boundary (dashed line). Positive residuals correspond to a more poleward motion than average. Plots are for CT boxes within $\pm 16^{\circ}$ of the central meridian and for CT boxes with $\left\langle\left|B_{\mathrm{c}}\right|\right\rangle$ lower than $20 \mathrm{G}$.

The equatorial rate $A_{\text {eq }}$ is also shown and is slightly larger inside $\mathrm{CHs}$ than outside $\mathrm{CHs}$ and at the $\mathrm{CH}$ boundary. More interesting is the fact that the photospheric rotation in $\mathrm{CHs}$ is consistently more differential than outside $\mathrm{CHs}$, with significantly more differential rotation at the boundaries compared to inside $\mathrm{CHs}$. The differences between the various categories are shown in Fig. 10 (bottom). At low latitudes, the differences are hardly significant. At latitudes greater than $40^{\circ}$ however, the differential rotation is not as strong inside $\mathrm{CHs}$ and outside $\mathrm{CHs}$ as at $\mathrm{CH}$ boundaries, as could already be suspected from the $A_{2}$ and $A_{4}$ coefficients in Table 2.

The antisymmetry between hemisphere, characterized by the $A_{1}$ and $A_{3}$ coefficients is also shown in Fig. 10 (top). In Meunier (2005a), it was found to be very small. Here it remains small outside $\mathrm{CHs}$ but it is significantly different from zero inside $\mathrm{CHs}$, as the Southern hemisphere rotates faster than the Northern hemisphere inside CHs. This is true at high latitude only (i.e. above $40^{\circ}$ ).

As for meridional circulation, the temporal variation in rotation residuals is computed separately at high and low latitudes, and in the two hemispheres. It is difficult to extract any systematic pattern. At low latitude, the rotation seems lower in CHs than outside CHs in 2001-2002 in the Northern hemisphere, and at the beginning of the cycle in the Southern hemisphere but the errorbars are quite large. At high latitude, no systematic pattern is seen either.

Figure 11 shows the variation in the CT rotation residuals versus $\mathrm{CH}$ area. For small $\mathrm{CHs}$, which correspond mostly to low latitudes, the residuals may be larger in $\mathrm{CHs}$ compared to outside, and similar in both hemispheres, but this is not significant. On the other hand, for $\mathrm{CH}$ sizes in the range of $6-9 \%$ of the visible hemisphere, the residuals are much larger in the Southern hemisphere than in the Northern hemisphere, which reproduces the asymmetry at high latitude obtained from the 
Table 2. Coefficients $A_{i}$ with $i=[0, \ldots, 4]$ from fits with Legendre polynomials in $\sin ^{i}(\theta)$ on rotation rates versus latitude (in deg/day) and equatorial rate, $A_{\text {eq }}$, from a polynomial fit in $\sin ^{i}(\theta)$.

\begin{tabular}{ccccccc}
\hline \hline Location & $A_{0}$ & $A_{1}$ & $A_{2}$ & $A_{3}$ & $A_{4}$ & $A_{\mathrm{eq}}$ \\
\hline Outside CHs & $13.309 \pm 0.002$ & $-0.004 \pm 0.003$ & $-2.559 \pm 0.006$ & $0.003 \pm 0.004$ & $-0.540 \pm 0.005$ & $14.386 \pm 0.001$ \\
Inside CHs & $13.317 \pm 0.006$ & $-0.060 \pm 0.013$ & $-2.589 \pm 0.019$ & $-0.067 \pm 0.019$ & $-0.553 \pm 0.023$ & $14.404 \pm 0.008$ \\
CH boundary & $13.281 \pm 0.004$ & $-0.010 \pm 0.007$ & $-2.682 \pm 0.009$ & $0.010 \pm 0.011$ & $-0.642 \pm 0.013$ & $14.381 \pm 0.008$ \\
\hline
\end{tabular}
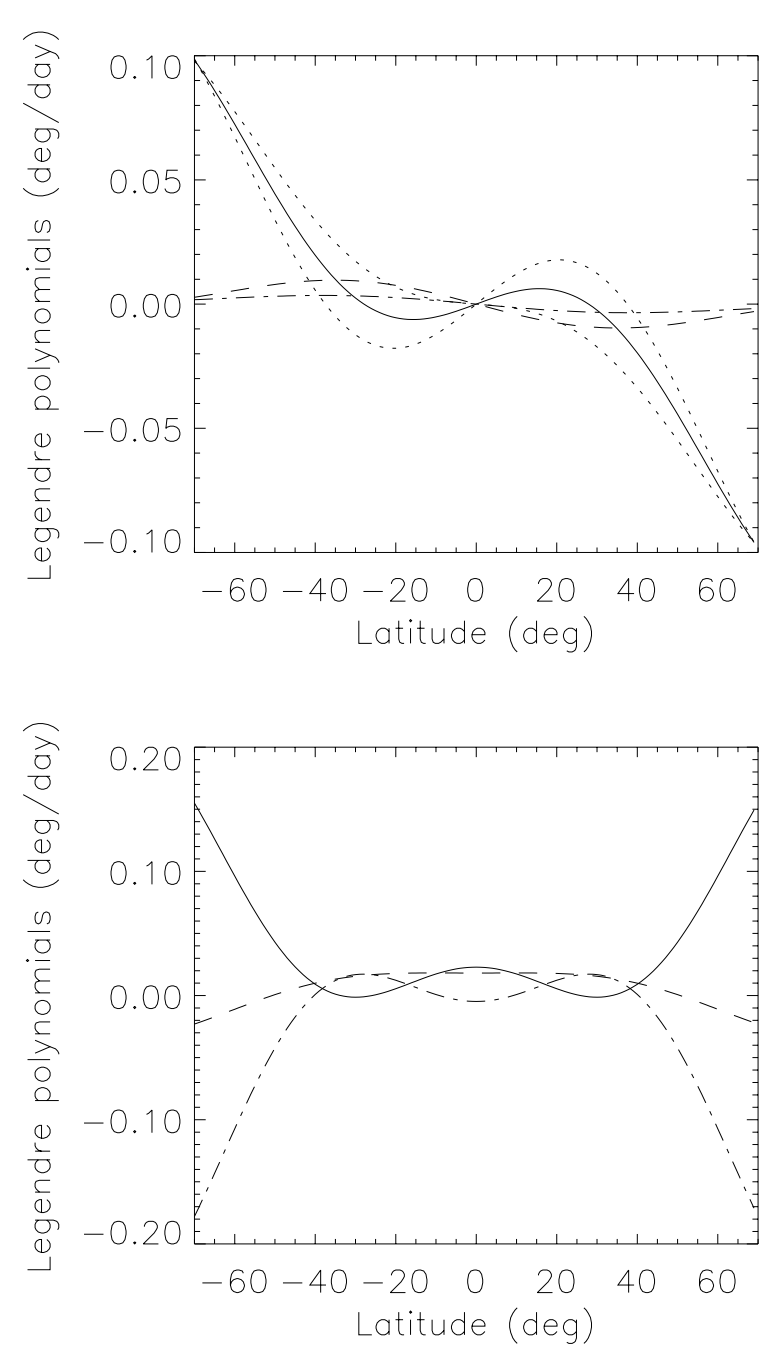

Fig. 10. Top: antisymmetrical part of the Legendre polynomial fit outside $\mathrm{CHs}$ (dotted-dashed line), inside $\mathrm{CHs}$ (solid line) and at $\mathrm{CH}$ boundary (dashed line), as deduced from the $A_{1}$ and $A_{3}$ coefficients in Table 2. The two dotted lines give an estimation of the uncertainty on the dashed line. Bottom: difference between the symmetrical part of the Legendre polynomial fit from the $A_{0}, A_{2}$, and $A_{4}$ coefficients in Table 2, in three cases, inside CHs minus outside CHs (dashed line), $\mathrm{CH}$ boundary minus outside $\mathrm{CHs}$ (dotted-dashed line), and inside $\mathrm{CHs}$ minus $\mathrm{CH}$ boundary (solid line). The errorbars are not shown for clarity, but are of the order of the signal at low latitude.

polynomial fits. This effect may still be present for the largest $\mathrm{CHs}$. This is also consistent with the fact that $\mathrm{CHs}$ in that size range are predominantly present at high latitude.

When separating the leading and following polarity regions as in Meunier (2005b), we find a similar result, with the following regions rotating faster than the leading regions for weak

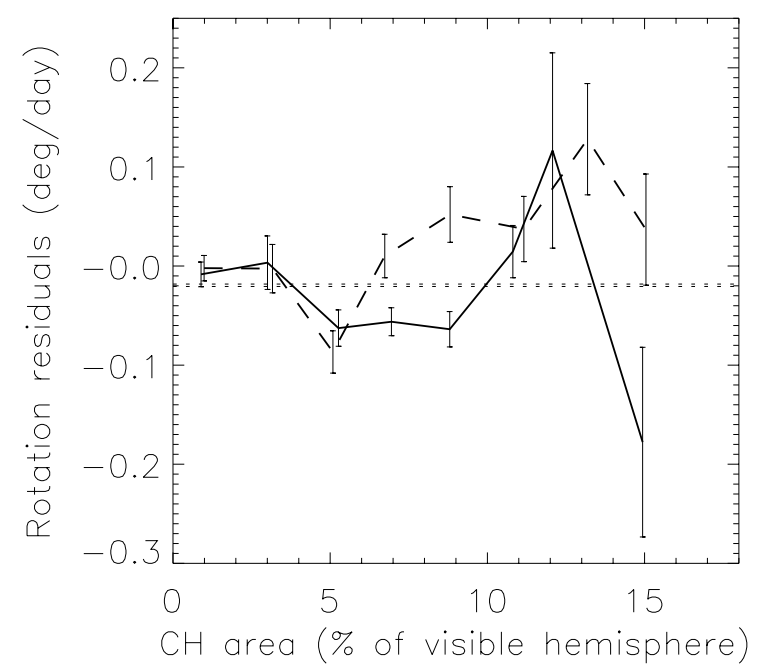

Fig. 11. Top: rotation residuals versus $\mathrm{CH}$ size (in percentage of visible hemisphere) in the Northern hemisphere (solid line) and Southern hemisphere (dashed line). The horizontal dotted line shows the average residual values outside CHs. Plots are for CT boxes within $\pm 16^{\circ}$ of the central meridian and for CT boxes with $\left\langle\left|B_{\mathrm{c}}\right|\right\rangle$ lower than $20 \mathrm{G}$.

fields. On the other hand, the variation in rotation with increasing $\left\langle\left|B_{\mathrm{c}}\right|\right\rangle$ observed by Meunier (2005a) is computed separately for the different categories. The difference between the various categories is not very significant.

\subsection{Velocity dispersions}

A Gaussian fit to the residual velocity distribution is performed in each latitude bin for the various categories. The latitude bins are $7.68^{\circ}$ wide, which is derived from the size of the CT boxes (see Sect. 3.1). The resulting HWHM are shown in Fig. 12 for $\mathrm{CHs}$ in the activity belt. For CT, the HWHM is smaller in $\mathrm{CHs}$ than outside $\mathrm{CHs}$ for both rotation and meridional circulation. The latitude dependence for both velocities is however different, with a peak around the equator for the rotation. The amplitude of this peak is twice as large in $\mathrm{CHs}$ than outside $\mathrm{CHs}$. On the other hand, the HWHM for the FT residuals tends to be larger in CHs than outside CHs. It is not as significant at the $\mathrm{CH}$ boundary, however. The latitude dependence is also different between rotation and meridional circulation, and is different to the CT variation.

\section{Discussion and conclusion}

Comparison of the large-scale dynamics deduced from crosscorrelation between pairs of photospheric magnetograms and 

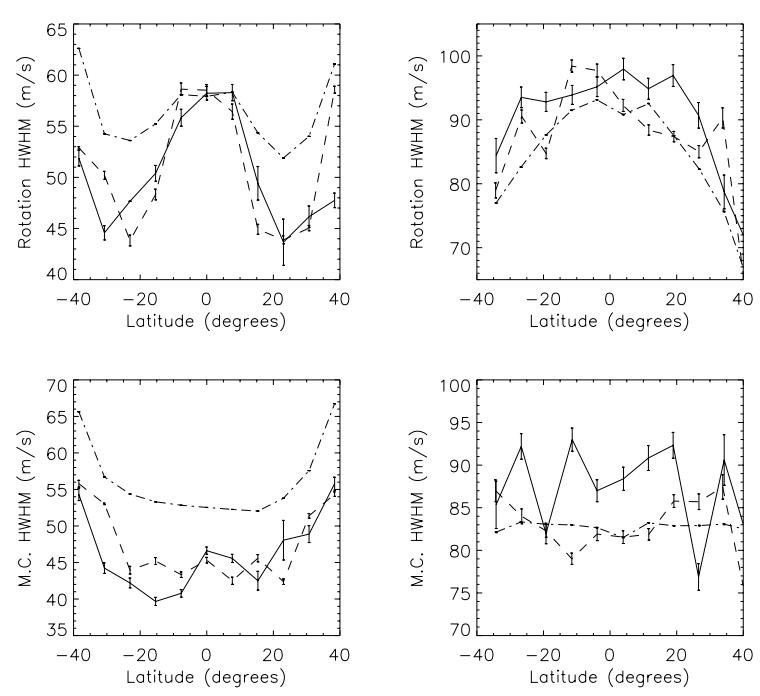

Fig. 12. Top: HWHM computed from gaussian fits over the CT (left) and FT (right) rotation residuals versus latitude, for boxes outside $\mathrm{CHs}$ (dotted-dashed lines), inside $\mathrm{CHs}$ (solid lines) and at the boundary (dashed lines). Bottom: same for meridional circulation. Plots are for CT boxes within $\pm 16^{\circ}$ of the central meridian and for $\left\langle\left|B_{\mathrm{c}}\right|\right\rangle$ lower than $20 \mathrm{G}$ and for FT structures smaller than $100 \mathrm{Mm}^{2}$ within $\pm 16^{\circ}$ of the central meridian.

$\mathrm{CH}$ data shows some significant differences between the behavior of the photosphere outside and inside CHs. However, the FT technique did not lead to many significant differences between $\mathrm{CHs}$ and outside $\mathrm{CHs}$, due to larger uncertainties. The new results can be summarized as follows:

- A weaker CT meridional circulation inside CHs, with a stronger converging pattern in $\mathrm{CHs}$ in the activity belt.

- A more differential CT rotation inside CHs at high latitude.

- More asymmetrical dynamics between the hemispheres for high latitude $\mathrm{CHs}$.

- A dependence of the dynamics on the $\mathrm{CH}$ size related to the latitude distribution of $\mathrm{CHs}$ and to the size distributions of CHs.

- A different temporal variability of the meridional circulation at high latitude.

- A smaller CT dispersion in velocity inside CHs in the activity belt than outside $\mathrm{CHs}$, and a larger FT dispersion.

- A different CT rotation in the core of the CHs compared to the boundaries, at high latitude.

- A different dependence of the meridional circulation on magnetic field, with steeper variations in $\mathrm{CHs}$ than outside for the weakest magnetic fields.

With supergranular diffusion, the differential rotation and meridional circulation are crucial ingredients in the distribution of the magnetic flux at the solar surface (Wang \& Sheeley 1990, for example). The more differential rotation in particular may appear in apparent contradiction to the relatively rigid rotation of CHs themselves. On the other hand, a weaker meridional circulation is compatible with a more differential rotation, which may explain the observed dynamics at high latitude. The variability of the dynamics inside $\mathrm{CHs}$ is as large as outside $\mathrm{CHs}$, while the average magnetic field level is less variable in our sample. The North-South asymmetry is already well known, as shown by the asymmetric distribution in size with latitude, but it also shows up in the dynamics inside $\mathrm{CHs}$, as the rotation is larger and the meridional circulation smaller in the Southern hemisphere at high latitude. These properties remain to be explained.

The dispersion in CT meridional circulation and rotation are mostly related to large-scale flows such as those derived by Ambrož (2001). They have rms amplitudes around 40-60 m/s while the flows derived by Ambrož (2001) have a rms amplitude around $40 \mathrm{~m} / \mathrm{s}$, so the orders of magnitudes are in very good agreement. This large-scale circulation seems therefore partially inhibited inside $\mathrm{CHs}$ and at the $\mathrm{CH}$ boundary. On the other hand, FT dispersions are related to the large-scale circulation as well, possibly at a different depth and therefore of different amplitude, but also to the diffusion of individual magnetic features. The FT dispersion is indeed larger than the CT ones, and is also larger inside $\mathrm{CHs}$ than outside $\mathrm{CHs}$. This suggests that the diffusion inside $\mathrm{CHs}$ and at $\mathrm{CH}$ boundary is larger than outside CHs. However, the FT dispersion is smaller than expected from typical diffusion coefficients obtained in the literature. For example, Berger et al. (1998) and Hagenaar et al. (1999) measured diffusion coefficients of about $50-70 \mathrm{~km}^{2} / \mathrm{s}$. Over $96 \mathrm{~min}$, this corresponds to an average displacement of the order of $180-220 \mathrm{~m} / \mathrm{s}$, which is larger than the FT dispersion obtained here, of the order of $130 \mathrm{~m} / \mathrm{s}$ at the equator. A value of $130 \mathrm{~m} / \mathrm{s}$ corresponds to a diffusion coefficient of about $25 \mathrm{~km}^{2} / \mathrm{s}$. This may be due to the fact that the FT tracking is biased toward larger network structures than in the work of Berger et al. (1998) and Hagenaar et al. (1999), as only structures with an unsigned magnetic field above $40 \mathrm{G}$ on MDI full-disk magnetograms and sizes larger than $10 \mathrm{Mm}^{2}$ are considered with FT. Therefore a more detailed analysis with high spatial resolution observations would be necessary.

Wang \& Sheeley (2002) explain the rigid rotation of $\mathrm{CHs}$ by some reconnection between small magnetic structures at the boundary of CHs. The field lines associated with these structures change their status between open and closed loops in the process and, therefore, cross the boundary, allowing for a rigid rotation of $\mathrm{CHs}$ despite the differential rotation of these magnetic structures. On the other hand, Fisk et al. (1999) explain the rigid rotation by large excursions of the field lines in latitude, due to the differential rotation and the non radial expansion of the solar wind from the Sun. The model of Wang \& Sheeley (2002) is already favored by the work of Madjarska et al. (2004), who found some evidence of reconnection along $\mathrm{CH}$ boundaries for the first time (these reconnections have been detected as bidirectional jets observed with SUMER on SOHO). Raju et al. (2005) also found some evidence of reconnection from the study of correlations between $\mathrm{CHs}$ and quiet Sun intensities. The results obtained here are compatible with a larger diffusion at the $\mathrm{CH}$ boundary compared to outside, but it is not larger than inside $\mathrm{CHs}$ for example. This larger diffusion may contribute to the larger excursion of the field lines as in the Fisk et al. (1999) model. On the other hand, this larger diffusion may be due to the open field-line of these structures, or to different properties of supergranules in CHs compared to outside CHs. This should be taken into account when 
studying the behavior of the open magnetic field, as in the model of Fisk \& Schwadron (2001). The main specific behavior of the $\mathrm{CH}$ boundary found here is the larger differential rotation at the $\mathrm{CH}$ boundary at high latitude. This may also play a role in the reconnection processes occuring at these boundaries.

These new results show that large-scale flows inside $\mathrm{CHs}$ are smaller than outside CHs. Despite the more differential rotation of the photosphere inside $\mathrm{CHs}$, these weaker flows may play a role in the processes leading to the rigid rotation of $\mathrm{CHs}$, while the small-scale dynamics may be consistent with reconnection processes. There is a possibility that the rigid boundary of CHs may prevent the largest scale flows from developing. This should be investigated further, as should the depth dependence of these characteristics. The temporal variability in the dynamics inside CHs must also be compared to the results obtained in Meunier (2005c). The large variation in meridional circulation of high-latitude $\mathrm{CHs}$ in the Southern hemisphere may be responsible for most of the variation in the Southern hemisphere observed by Meunier et al. (2005c), but that does not seem to be the case in the Northern hemisphere. In future works, it will be necessary to increase the temporal coverage (by using several cycles), as with one cycle the uncertainties are still large when trying to study the dependence on the various physical parameters.

Acknowledgements. SOHO is a mission of international cooperation between the European Space Agency (ESA) and NASA. The coronal hole data used in this work were compiled by K. Harvey and F. Recely using NSO KPVT observations under an NSF grant. I am very grateful to C. Henney for his help with these data and to G. de Toma for her estimations of the magnetic contents of coronal holes.

\section{References}

Ambrož, P. 2001, Sol. Phys., 198, 253

Antonucci, E., Azzarelli, L., Casalini, P., Cerri, S., \& Denoth, F. 1979, Sol. Phys., 63, 17
Berger, T. E., Löfdahl, M. G., Shine, R. A., \& Title, A. M. 1998, ApJ, 506,439

Brajša, R., Wöhl, H., Vršnak, B., et al. 2004, A\&A, 414, 707

de Toma, G., \& Arge, N. 2005, 22th Sacramento Peak Solar Workshop, October 2004, in press

Fisk, L. A., Zurbuchen, T. H., \& Schwadron, N. A. 1999, ApJ, 521, 868

Fisk, L. A., \& Schwadron, N. A. 2001, ApJ, 560, 425

Haber, D. A., Hindman, B. W., \& Toomre, J. 2003, in Proc. SOHO 12/GONG+ 2002, Local and Global Helioseismology: The Present and Future, ed. H. Sawaya-Lacoste, 103

Hagenaar, H. J., Schrijver, C. J., Title, A. M., \& Shine, R. A. 1999, ApJ, 511, 932

Harvey, K. L., \& Recely, F. 2002, Sol. Phys., 211, 31

Harvey, K. L., Sheeley, N. R. Jr., \& Harvey, J. W. 1982, Sol. Phys., 79, 149

Kahler, S. W., \& Hudson, H. S. 2002, ApJ, 574, 467

Komm, R. W., Howard, R. F., \& Harvey, J. W. 1993a, Sol. Phys., 145, 1

Komm, R. W., Howard, R. F., \& Harvey, J. W. 1993b, Sol. Phys., 147, 203

Madjarska, M. S., Doyle, J. G., \& van Driel-Gesztelyi, L. 2004, ApJ, 603, L57

Meunier, N. 1999, ApJ, 527, 967

Meunier, N. 2003, A\&A, 405, 1107

Meunier, N. 2005a, A\&A, 436, 1075

Meunier, N. 2005b, A\&A, 437, 303

Meunier, N. 2005c, A\&A, 442, 693

Raju, K. P., Bromage, B. J. I., Chapman, S. A., \& Del Zanna, G. 2005, A\&A, 432, 341

Scherrer, P., Bogart, R. S., Bush, R. I., et al. 1995, Sol. Phys., 162, 129

Snodgrass, H. B., \& Ulrich, R. K. 1990, ApJ, 351, 309

Wang, H. 1988, Sol. Phys., 116, 1

Wang, Y.-M., \& Sheeley, N. R. Jr. 1990, ApJ, 365, 372

Wang, Y.-M., \& Sheeley, N. R. Jr. 2004, ApJ, 612, 1196

Wang, Y.-M., Hawley, S. H., \& Sheeley, N. R. Jr. 1996, Science, 271, 464

Zhao, J., \& Kosovichev, A. G. 2004, ApJ, 603, 776 